Cardiac arrhythmias affected five antenatally-diagnosed fetuses (56\%), with one requiring emergency delivery at 28 weeks and ongoing neonatal management.

The majority of cardiac rhabdomyomas in both groups were located in the ventricles. Tumour growth continued up to 28 weeks of age amongst all surviving children, followed by spontaneous regression, with no need for resective surgery. There was a high prevalence of neurological morbidity in both groups.

Conclusion Antenatal cardiac rhabdomyomas, occurring as part of the TSC, can cause significant morbidity, which is rarely fatal, but warrants careful monitoring until the point of tumour regression. The burden of neurological disease is high in children, compared with the largely favourable cardiac outcome.

\section{PF.57 FETAL MACROSOMIA: A RETROSPECTIVE OBSERVATIONAL STUDY}

doi:10.1136/archdischild-2013-303966.066

G Argentesi, KF Walker, S Raouf. Royal Derby Hospital, Derby, UK

Objectives Maternal obesity is one of the biggest challenges facing modern obstetrics. The focus of this study was to investigate whether speculation that fetal macrosomia may be on the rise as a consequence of rising levels of maternal obesity and to observe if there was an increase in complications as a result of fetal macrosomia, which is defined as a birth weight of $4.0 \mathrm{~kg}$ and above.

Method A retrospective observational study of all babies weighing $4.0 \mathrm{~kg}$ or more born in 2011 at Royal Derby Hospital. Data was collected on maternal parameters such as BMI, fasting glucose and glucose tolerance test, gestation at delivery, delivery outcomes, neonatal birth weight, Apgar scores and their overall outcome. The data was then compared to data from both 2001 and 1991 recovered from the hospital archives.

Results In 2011, 11.1\% of the total babies born that year had a birthweight of $\geq 4.0 \mathrm{~kg}$. In 2001, 10.3\% and in 1991, $10.7 \%$. The average BMI of women who gave birth to a baby weighing $\geq 4.0 \mathrm{~kg}$ in 2011 was 28.

Conclusion Although there is speculation that fetal macrosomia is on the rise, in association with gestational diabetes and a rise in maternal BMI, we found that over the last 20 years the number of macrosomic babies has not increased at the Royal Derby Hospital. The overall maternal BMI was only slightly higher than average and deliveries involving macrosomic babies were not complicated by a higher rate of caesareans sections or instrumental deliveries or obstetric complications.

\section{PF.58 MANAGEMENT AND OUTCOME OF VASA PRAEVIA: A TEN YEAR REVIEW}

doi:10.1136/archdischild-2013-303966.067

T Kubba, F Ushakov, S Cicero, G Attilakos. Department of Fetal Medicine, UCL and UCLH Institute for Women's Health, London, UK

Introduction Vasa Praevia (VP) describes fetal vessels coursing through the membranes over the internal os, unprotected by placental tissue or umbilical cord. VP is associated with significant fetal risk when membrane rupture occurs. The RCOG guideline on VP recommends antenatal admission from 28-32 weeks until delivery in a unit with appropriate neonatal facilities to facilitate quicker intervention in the event of bleeding or labour.

Aim To review the management and outcome of VP cases at a tertiary teaching hospital.

Methods We undertook a ten year retrospective review (2002 to 2012) of all cases of confirmed VP. Cases were identified using the discharge codes of all inpatient episodes and the fetal medicine unit database. We reviewed the ultrasound scans and notes of all cases.
Results We identified 15 confirmed cases of VP. 14 cases were diagnosed antenatally. The median GA at diagnosis was $25+3$ weeks. 9 cases were admitted antenatally (duration: 2 days to 5 weeks). None of the admitted cases went into labour.

$11 / 15$ cases had elective LSCS and 4/15 had emergency LSCS (2/4 had category 1 LSCS). The median GA at delivery was $37+3$ weeks. The single undiagnosed case resulted in neonatal death secondary to VP.

\section{Conclusions}

1. VP is a rare condition.

2. A high proportion of cases were diagnosed antenatally, however there may be cases which were never diagnosed and did not cause adverse events.

3. Further evidence is needed on the necessity and timing of antenatal admission.

\section{PF.59 WHAT IS THE OPTIMAL DOSE OF LOW MOLECULAR WEIGHT HEPARIN IN PREGNANT WOMEN WITH RAISED BMI?}

doi:10.1136/archdischild-2013-303966.068

'S Rahman, ${ }^{2 D}$ Stewart, 'L Byrd, 'C Tower. 'St. Mary's hospital, Manchester, UK; ${ }^{2}$ Newcastle university medical school, Newcastle, UK

Introduction Obesity is a risk factor for thromboembolism in pregnancy. Recent RCOG guidelines suggested a non-evidence based weight-dependent protocol in which monitoring of anti-Xa levels was not required. This contrasted with local guidelines which used BMI based dosing, using anti-Xa levels to determine correct dose with $\mathrm{BMI}>35$. We sought to investigate the impact of the different strategies in a cohort of women treated with antenatal thromboprophylaxis.

Methods We retrospectively audited the thromboprophylaxis practise amongst 42 women between September 2009 and September 2011. We observed tinzaparin dosing, frequency of anti Xa levels, dose changes, and pregnancy outcomes.

Results 39/42 (93\%) had a BMI over 35 and had anti-Xa measurements. Using the local protocol 15/39 (38\%) required dose increases and all patients received a higher dose than suggested by RCOG guidelines (median 3000 IU, interquartile range(1OR) 3000-5000 IU). There were no thrombotic events and 25/38 (66\%) achieved a vaginal delivery. The median estimated blood loss at delivery was $350 \mathrm{ml}$ (IOR 200-725 mls) and 3 women suffered a major PPH > $1500 \mathrm{mls}$.

Discussion All our patients received a higher tinzaparin dose than suggested by RCOG guidelines, but shown by anti-Xa monitoring to be therapeutic. In these small numbers, there was a high vaginal delivery rate $(66 \%)$ and although 3 women suffered a major $\mathrm{PPH}$, the median blood loss was within an acceptable range for this high risk population. Without appropriate monitoring, RCOG guidelines may be resulting in suboptimal anticoagulation in women with raised BMI, whilst exposing them to the risks and side effects of LMWH.

\section{PF.60 NEONATAL HYPOPHOSPHATASIA: A RARE DISORDER AND NEW TREATMENT}

doi:10.1136/archdischild-2013-303966.069

${ }^{1} \mathrm{~S}$ Hiremath, 'VK Devendra Kumar, ${ }^{2} \mathrm{R}$ Padidela, ${ }^{2 Z}$ Mughal. 'Lancashire Women 8 Newborn Centre, Burnley General Hospital, Burnley, UK; ${ }^{2}$ Central Manchester University Hospitals NHS Foundation Trust, Manchester, UK

Hypophosphatasia is a rare inborn error of metabolism resulting from mutations in the gene for the tissue-nonspecific isozyme of alkaline phosphatase (TNSALP). There is deficiency of alkaline phosphatase activity leading to severe rickets/osteomalacia. Severely affected babies die from respiratory insufficiency. There is no licenced medical treatment available. We report a case diagnosed 
recently with hypophosphatasia who is receiving pioneering enzyme replacement treatment.

Baby I was born at 34/40 and required ventilatory support from birth due to respiratory insufficiency. She was noted to have short limbs, hypotonia, and thin ribs on x-rays. Her serum alkaline phosphatase was low; urinary phosphoethanolamine and serum calcium were elevated confirming hypophosphatasia.

In a recent multinational study of 11 patients with severe hypophosphatasia, treatment with recombinant human bone targeted TNSALP (ENB 0040) has been shown to improve bone mineralization. This was associated with healing of rickets, improved developmental milestones and pulmonary function.

Under guidance from the regional Metabolic Bone team at Manchester and with parental consent, Baby I was commenced on ENB 0040 (Asfotase alfa) at the age of 4 weeks with subcutaneous injections three times a week. The drug is being offered to this infant on compassionate grounds by the manufacturer (Alexion pharmaceuticals).

Within 6 weeks of treatment calcium requirement of infant has increased and X-rays have demonstrated remarkable improvement in mineralisation. She remains ventilator dependant with a tracheostomy in situ but, we anticipate that with ENB 0040 treatment, improvement in bone mineralisation and muscle function will facilitate weaning from ventilation.

\section{PF.61 STARRY SKY PATTERN OF FETAL LIVER ASSOCIATED WITH TWIN ANAEMIA POLYCYTHAEMIA SEQUENCE IN MONOCHORIONIC TWINS}

doi:10.1136/archdischild-2013-303966.070

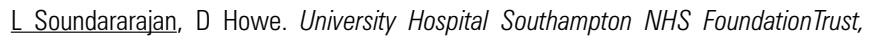
Southampton, UK

Twin anaemia-polycythaemia sequence (TAPS) is an atypical form of twin-twin transfusion syndrome (TTTS) that presents as a large inter-twin haemoglobin difference with one twin developing anaemia and the other developing polycythaemia, without oligohydramnios-polyhydramnios sequence. The prenatal diagnostic criteria for TAPS require that the middle cerebral artery-peak systolic velocity (MCA-PSV) measure greater than 1.5 multiples of median (MoM) in the anaemic twin and less than 0.8 MoM in the polycythaemic twin.

Starry-sky liver appearance was first described in 1980 by Kurtz et al, as corresponding to oedematous swelling of hepatocytes causing decreased echogenicity of the parenchyma (sky) as well as better visualisation of the fibrous walls of the portal vein (stars).

We present a case of monochorionic diamniotic twins with spontaneous twin anaemia- polycythaemia sequence (TAPS) in which the rare sonographic appearance of starry sky liver was seen in the recipient (polycythaemic) twin. The polycythaemic twin had starry sky liver appearance from 25 weeks gestation prior to any sonographic features of TAPS. She was delivered at 28 weeks 6 days gestation when diagnosis of TAPS was made, due to deterioration in growth and Dopplers. One twin was anaemic with haemoglobin of $10.5 \mathrm{~g} / \mathrm{dl}$ and the other polycythaemic with haemoglobin of $28.0 \mathrm{~g} / \mathrm{dl}$.

Conclusion In our case, starry sky appearance of the liver was the early sonographic feature of Twin anaemia polycythaemia sequence.

\section{PF.62 COMPUTATIONAL RECONSTRUCTION OF HUMAN FETAL CARDIAC VENTRICULAR WALL DEVELOPMENT}

doi:10.1136/archdischild-2013-303966.071

'E Pervolaraki, ${ }^{2} \mathrm{R}$ Anderson, ${ }^{3} \mathrm{M}$ Paley, ${ }^{1} \mathrm{AV}$ Holden. 'Faculty of Biological Sciences, University of Leeds, Leeds, UK; ${ }^{2}$ MRC Centre for Reproductive Health, University of Edinburgh, Edinburgh, UK; ${ }^{3}$ Academic Radiology, University of Sheffield, Sheffield, UK
We construct a computational model of the electrophysiology of the developing heart.

Anisotropic geometry at $\sim 100 \mu \mathrm{m}$ voxel resolution was obtained from Diffusion Tensor and Fast Low Angle Shot Magnetic Resonance Imaging and electrical activity from fetal electrocardiograms obtained longitudinally from one gestation and from multiple studies.

Transmural myofibre organisation is established by 136 days gestational age (DGA) and during $2^{\text {nd }}$ and $3^{\text {rd }}$ trimester $\mathrm{OR}$ intervals decrease by $20 \mathrm{~ms}$ while heart dimensions increase by a 2 -fold. This implies an increase in the ventricular conduction velocity. A computational model of the 140 DGA human ventricle is presented that combines cell electrophysiology with anisotropic geometry.

\section{PF.63 A RARE CASE OF FETAL ANAEMIA DUE TO CONGENITAL PYROPOIKILOCYTOSIS TREATED BY INTRAUTERINE FETAL BLOOD TRANSFUSION}

doi:10.1136/archdischild-2013-303966.072

${ }^{1}$ K Lim, ${ }^{1}$ JEAK Bamfo, 'ED Johnstone, ${ }^{2} \mathrm{~A}$ Will. ${ }^{1}$ Department of Obstetrics and Gynaecology, St Mary's Hospital, Mancheseter, UK; ${ }^{2}$ Department of Paediatric Haematology, Central Manchester University Hospital, Manchester, UK

We present the first case of a pre-natal diagnosis of fetal anaemia due to congenital pyropoikilocytosis treated with intrauterine fetal blood transfusion.

A 31 year old woman of Caucasian origin was referred to the fetal medicine unit at 29 weeks gestation with suspected fetal anaemia. She had 3 previous miscarriages and delivered a term female infant who was severely jaundiced and anaemic at birth, requiring multiple exchange transfusions. That infant was diagnosed with congenital pyropoikilocytosis.

In this pregnancy, a middle cerebral artery Doppler peak systolic velocity (MCA PSV) performed at 26 and 28 weeks gestation suggested mild to moderate fetal anaemia. At 29 weeks, MCA PSV indicated severe fetal anaemia. There were no signs of hydrops fetalis. Fetal blood sampling confirmed fetal anaemia and fetal blood transfusion was performed. Fetal blood film confirmed congenital pyropoikilocytosis. At 31 weeks, a repeat fetal blood transfusion was indicated but was unsuccessful due to transient fetal bradycardia. Delivery was prompted and at 32 weeks, a female infant was delivered by elective caesarean section. The infant was anaemic requiring multiple exchange transfusions. Neonatal recovery was uneventful.

Congenital pyropoikilocytosis is an autosomal recessive rare hemolytic anaemia due to an erythocyte membrane defect. It is more often seen in black populations and has rarely been seen in white European populations. Doppler prediction of fetal anaemia using MCA PSV should be advocated in women whose previous pregnancies show them to be at high risk of recurrent fetal or neonatal hemolytic anaemias due to rare erythrocyte defects.

\section{PF.64 COMPLICATED SEQUELAE OF PARVOVIRUS AFFECTED PREGNANCIES}

doi:10.1136/archdischild-2013-303966.073

K Flood, N Burke, S Muellers, FD Malone. Royal College of Surgeons in Ireland, Dublin, Ireland

During the recent epidemic of Parvovirus infection, three complicated pregnancies were managed in the Rotunda Hospital. The fetuses were significantly affected in all three cases, presenting with ultrasonographic findings consistent with severe anaemia; all required intra-uterine fetal transfusions.

Case 1: The first case involved a 30 year old multip who presented at 20 weeks with severe fetal hydrops and a history of Parvovirus 